\title{
Incidence and Risk Factors of Postoperative Delirium in Cervical Spine Surgery
}

\author{
Takahiro Ushida, MD, PhD, ${ }^{*} † \ddagger$ Takeshi Yokoyama, MD, PhD, $\$$ Yasuyo Kishida, BSN, १ \\ Mika Hosokawa, BSN, ๆ Shinichirou Taniguchi, MD, PhD, ${ }^{*} \ddagger$ Shinsuke Inoue, MD, PhD, ${ }^{*}$ \\ Ryuichi Takemasa, MD, PhD, ${ }^{*}$ Katsutoshi Suetomi, PhD, $\neq$ Young-Chang P. Arai, MD, PhD, † \\ Matthew McLaughlin, MA, $\ddagger$ and Toshikazu Tani, MD, PhD*
}

Study Design. Retrospective clinical review and prospective report of postoperative delirium after cervical spine surgeries.

Objective. To investigate factors contributing to the development of delirium after cervical surgery and see whether amended therapeutic protocols could improve or alter postoperative outcomes.

Summary of Background Data. Important consequences of postoperative delirium for the orthopedic patients include impaired recovery and increased morbidity and mortality. Although its risk factors have been reported in orthopedic surgery, there are a very few reports regarding postoperative delirium in spine surgery.

Methods. Eighty-one cervical myelopathy patients were retrospectively examined about the incidence of postoperative delirium and the risk factors. Similarly, 41 patients who received postoperative care under modified protocols were prospectively examined.

Results. Postoperative delirium occurred more commonly in patients over 70 years and those with hearing impairment. Patients who received high-dose methylprednisolone (>1000 $\mathrm{mg}$ ) demonstrated an increased incidence of postoperative delirium. Under modified protocol, we reduced the usage of methylprednisolone and encouraged free body movement with cervical orthosis immediately after surgery. The incidence of postoperative delirium was significantly lower under the modified protocol.

Conclusion. Early commencement of mobilization after cervical spine surgery would be crucial to the prevention of postoperative delirium in the elderly.

Key words: postoperative delirium, cervical spine surgery, hearing impairments, methylprednisolone. Spine 2009;34: 2500-2504

From the *Department of Orthopaedic Surgery, Kochi Medical School, Kochi, Japan; †Multidisciplinary Pain Center, Aichi Medical University, Aichi, Japan; ‡Nankoku Pain Research Group, Kochi Medical School, Kochi, Japan; \$Department of Anesthesiology, Kochi Medical School, Kochi, Japan; and IINursing Unit of Kochi Medical School Hospital, Kochi, Japan.

Acknowledgment date: January 5, 2009. Revision date: March 25, 2009. Acceptance date: April 7, 2009.

All study protocols were approved by the Research committee of Kochi Medical School Hospital.

The device(s)/drug(s) is/are FDA-approved or approved by corresponding national agency for this indication.

No funds were received in support of this work. No benefits in any form have been or will be received from a commercial party related directly or indirectly to the subject of this manuscript.

Address correspondence and reprint requests to Takahiro Ushida, MD, $\mathrm{PhD}$, Multidisciplinary Pain Center, Aichi Medical University, Karimata 21, Yazako, Nagakute-cho, 480-1195 Aichi, Japan; E-mail: ushidat-koc@umin.ac.jp
Delirium is an acute and relatively sudden decline in attention-focus, perception, and cognition and known to occur usually in the elderly people. ${ }^{1}$ Its incidence is known to increase during the perioperative period and it can cause major medical management problems. Several previous reviews indicate that delirium may affect a large proportion of orthopedic patients and report prevalence rates of $28 \%$ to $61 \%$. ${ }^{2,3}$ Although the incidence in hip and knee joint surgery has been widely reported, there are few studies regarding postoperative delirium in spine surgery. ${ }^{4,5}$

Increasing age, blood urea levels, cardiothoracic index, hypertension, smoking habits, blood replacement during bypass, atrial fibrillation (AF), pneumonia, and blood fluid balance in the postoperative period were found to be significant risk factors for delirium by logistic regression analysis. ${ }^{6}$ Previous reports also suggest that prior cognitive impairment and reduced hemoglobin and hematocrit levels are significant risk factors for postoperative delirium. With the development of modern medicine, the percentage of elderly people in the population is increasing and thus the incidence of postoperative delirium would increase. Spinal surgery in general and cervical spinal surgery in particular entail specific management problems and risk factors. During the postoperative phase of cervical spine surgery, it is essential to maintain spinal immobilization for preventing spinal cord trauma and improving neurologic outcomes.

The aim of the present study was to investigate factors contributing to the development of postoperative delirium in cervical surgery. We also investigated whether amended therapeutic protocols could improve or alter the incidence of postoperative delirium.

\section{- Materials and Methods}

\section{Methods}

This study was undertaken during 2003-2007 by analyzing perioperative delirium in elderly patients with cervical myelopathy (age, $>50$ years) in Kochi Medical School Hospital. Surgical procedures and postoperative treatment protocols used in the study were based on institutional policy and clinical guidelines for cervical surgery. In 2006, clinical management guidelines were modified as a result of the preliminary findings in this study. All study protocols were approved by the Research Committee of Kochi Medical School Hospital.

\section{Participants}

All admitted patients who passed indication criteria for cervical decompression surgery were interviewed and examined to rule 
out the pre-existence of dementia or other psychological disorders. Dementia was excluded by assessment using the revised Hasegawa Dementia Scale. ${ }^{7}$ After primary interview, 81 cervical myelopathy patients (age, 53- 84 years; average, 69.8 years) were included in this study as group 1 patients. These 81 patients (group 1) underwent surgery during 2003 to 2006. Similarly, 41 patients (52-86 years; average, 68.1 year), who underwent postoperative care under modified protocols were included in group 2 (2006-2008). Primary spine diseases in group 1 were as follows: ossification of posterior longitudinal ligament (OPLL) (10 cases), cervical spondylotic myelopathy (50 cases), cervical disc herniation (6 cases), atlantoaxial subluxation (6 cases), and others (9 cases). Group 2 disease conditions were OPLL (2 cases), cervical spondylotic myelopathy (29 cases), and cervical disc herniation (10 cases).

Preoperative planning was conducted using standardized institutional policy in the following cases: anterior cervical decompression/fusion (61 cases), laminoplasty (29 cases), and posterior fusion (14 cases).

Additional nursing care checks on mental status were made by assessment of visual and auditory function. Hearing impairment was diagnosed when patients had difficulty repeating words spoken in a conversation voice by the head nurse standing behind the patients in a silent room in order to prevent lip-read.

\section{Perioperative Routine Care}

For all participants, general anesthesia was induced with propofol and maintained by sevoflurane and nitrous oxide inhalation methods. During surgery, the patients were mechanically ventilated with $67 \%$ nitrous oxide and $1.5 \%$ to $2 \%$ end-tidal sevoflurane in oxygen while end-tidal carbon dioxide was maintained at 35 to $38 \mathrm{~mm} \mathrm{Hg}$. With group 1 participants, methylprednisolone $(500 \mathrm{mg}$ ) was administered before undergoing surgical disruption of the epidural space and ligaments (posterior longitudinal ligament or ligamentum flavum). With both groups, additional methylprednisolone $(\sim 2000 \mathrm{mg})$ was administered if neurologic symptoms developed in the early postoperative phase (first or second day). At postoperative day 2 , the drainage tube was removed from the cervical operative wound.

The intravenous infusion line was maintained until postoperative day 3 and antibiotics were administered. During the early postoperative phase, group 1 patients were instructed to avoid voluntary body and neck movement. Pads were placed on both sides of the head. For group 2 patients, however, a Philadelphia-type neck orthosis was fitted at postoperative day 1 , and patients were encouraged to move and change posture on the bed.

At postoperative day 3, cervical orthosis (Philadelphia-type collar) was applied to immobilize the cervical spine (for group 1 patients) and patients were allowed to move by walker or wheel chair in accordance with general medical and psychological condition.

If necessary, cervical magnetic resonance imaging (MRI) was performed to check bone alignment and to exclude residual spinal cord compression or edema.

During the perioperative period, general medical observations (body temperature, $\mathrm{O}_{2}$ saturation, pain, sleep disruption, and psychological status) were recorded. The degree of postoperative pain was evaluated by use of a numerical pain scale and recorded doses of analgesics. Sleep disruption was evaluated by interview and usage of narcoleptics.

\section{Psychological Evaluations}

Clinical features of delirium were evaluated by nurses. Observations of subjects was conducted each shift and recorded using the delirium observation screening (DOS) that is developed on the basis of the Diagnostic and Statistical Manual of Mental Disorders, Fourth Edition (DSM-IV) diagnostic criteria. ${ }^{4,8}$ The DOS scale is with 13 items that can be rated as present or absent in less than 5 minutes. The highest total score is 13 . Three or more points indicate a delirium. A diagnosis of delirium was made by 2 spinal surgeons (S.I.T., T.U.).

\section{Survey of Perioperative Conditions}

In addition to estimates of hearing and visual functions, other individual factors (age, pre-existing disease), surgery-related factors (surgical procedure time, methylprednisolone dosage, duration of anesthesia, body temperature, pain, dyssomnia) and perioperative circumstances (usage of intensive care unit [ICU], number of drain tubes, and infusion lines, personal family attendances) were investigated in both group 1 and 2 .

\section{Statistical Analysis}

In each group, the incidence of delirium and possible risk factors (individual factors, therapeutic factors, and perioperative circumstances) were analyzed by Fisher exact test. If the factors have 3 or more classifications, $\chi^{2}$ test was underwent for first level analysis and Fisher exact test was followed for further analysis.

Occurrence rates of postoperative delirium in group 1 and 2 were analyzed by Fisher exact test to clarify the efficacy of modified perioperative protocols.

\section{- Results}

\section{Group 1 Results}

Incidence of Postoperative Delirium Versus Patient Age. No patient in group 1 less than 60 years developed postcervical surgery delirium regardless of other profiles. The incidence of postcervical surgery delirium increased in patients over 70 years with rates of $30 \%$ or more (Figure 1 ).

Incidence of Postoperative Delirium Versus Hearing Impairment. Delirium incidence rates in group 1 participants with no hearing impairment were compared with those with auditory deficits to assess the influence of hearing problems on postoperative delirium outcomes. Fifty percent of patients with hearing impairment in normal daily

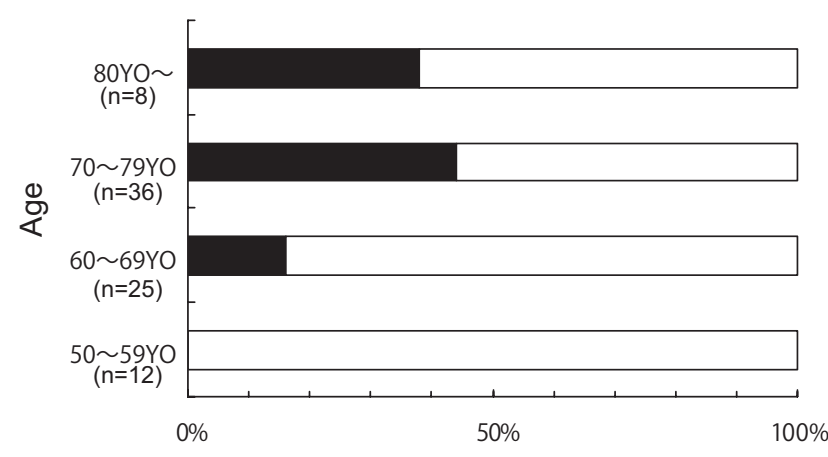

Incidence of post-cervical spine surgery delirium

Figure 1. Age differences in incidence of postcervical spine surgery delirium in group 1 patients. Age 70 years or older showed significantly increased incidence than younger ages. 


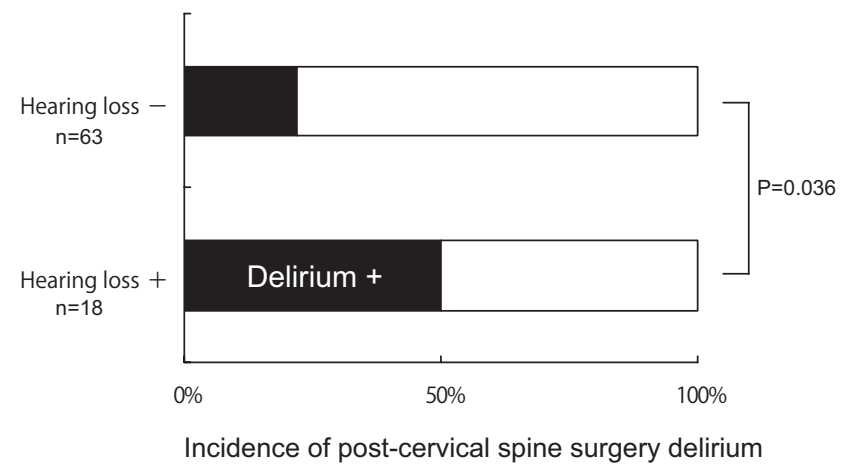

Figure 2. Incidence of postcervical spine surgery delirium versus hearing impairment in group 1. Patients with hearing impairment showed significantly increased incident rate than patients without hearing impairment in group $1(P=0.036)$.

life activity, experienced postoperative delirium while the incidence was only $22 \%$ when hearing was normal (Figure 2).

Other individual factors such as types of primary spinal disease, coincidental medical conditions (diabetes, cerebrovascular disease, etc.) had no influence on the incidence of postoperative delirium.

Incidence of Postoperative Delirium Versus Therapy Associated Factors. Types of surgical procedure, duration of anesthesia, postoperative body temperature, $\mathrm{O}_{2}$ saturations (postoperative), perioperative pain intensity, dyssomnia, and usage of analgesics or anxiolytics had no significant effect on the incidence of postoperative delirium. However, the use of high-dose methylprednisolone $(>1000$ $\mathrm{mg}$ ), administered for prevention of spinal cord edema, was associated with increased occurrence rates for this condition (Figure 3).

Postoperative Delirium Versus Perioperative Circumstances. Postoperative ICU treatment and number of drain tubes and infusion lines had no association with the incidence of postoperative delirium. Additionally, the attendance and support of family members, which may strongly influence the psychological stability of perioperative pa-

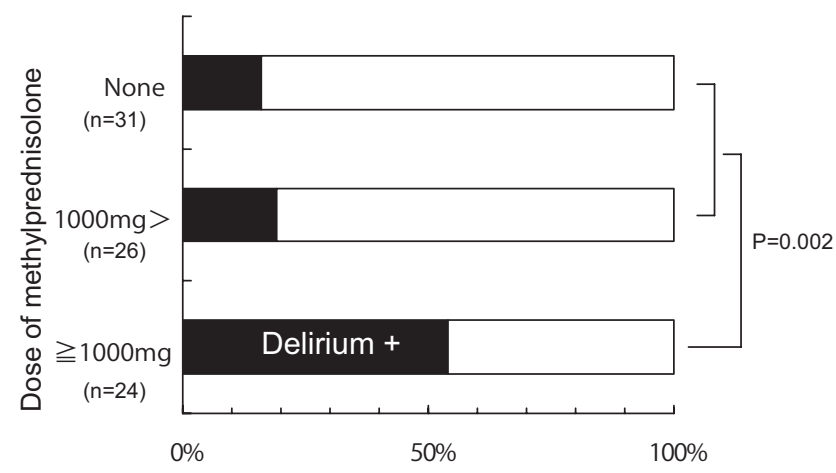

Incidence of post-cervical spine surgery delirium

Figure 3. Incidence of postcervical spine surgery delirium versus usage of methylprednisolone in group 1. Patients who used methylprednisolone ( $>1000 \mathrm{mg}$ ) showed significantly increased in incidence of postcervical spine surgery delirium in group $1(P=0.002)$.
Table 1. Group Comparison of Incidence of Postoperative Delirium

\begin{tabular}{lcr}
\hline & \multicolumn{2}{c}{ Delirium } \\
\cline { 2 - 3 } Treatment Protocol & + & \\
\hline $\begin{array}{l}\text { First protocol } \\
\quad 2003-2006(\mathrm{n}=81)\end{array}$ & $23^{*}$ & 58 \\
$\begin{array}{l}\text { Modified protocol } \\
2006-2008(\mathrm{n}=41)\end{array}$ & 3 & 38 \\
${ }^{*} P=0.0091$. & & \\
\hline
\end{tabular}

tients, had no statistical association with the incidence of delirium.

\section{Group 2 Results}

In group 2, postoperative delirium occurred in only 3 patients who were more than 70 years. Individual factors such as types of spinal disease or medical coconditions (diabetes, cerebrovascular disease, etc.) were found to have no effect on the incidence of postoperative delirium. Types of surgical procedure, duration of anesthesia, postoperative body temperature, $\mathrm{O}_{2}$ saturations, perioperative pain intensity, dyssomnia, and usage of analgesics or anxiolytics were not associated with the incidence of postoperative delirium. Postoperative ICU treatment, number of drain tubes, infusion lines, and the presence of supporting family members also had no effect on the incidence of postoperative delirium. In contrast to group 1 , usage of high-dose methylprednisolone had no observed effect on postoperative outcomes.

\section{Group Comparison}

Among 81 in group 1 patients, 23 patients became postoperative delirium and only 3 patients among 41 in group 2 patients. In accordance with the statistical analysis, incidence of postoperative delirium was significantly differed $(P=0.0091)$. (Table 1$)$ In all delirium cases, symptoms improved soon after commencement of mobilization by removal of tubing, ambulation, and sleeping with cervical orthosis.

\section{Discussion}

In the present study, postoperative delirium occurred more frequently in patients over 70 years, patients with hearing impairment, and patients who had received highdose methylprednisolone under early protocols. Aging is a well-known risk factor in postoperative delirium development. In line with our results, Kawaguchi et al observed postspinal surgery delirium only in patients in their 70 s or 80 s, suggesting that special attention must be paid to subjects aged over 70 concerning the occurrence of delirium. ${ }^{5}$

Delirium symptoms improved soon after resumption of normal circadian rhythm by removal of tubing, ambulation, and sleeping with cervical orthosis. Interestingly, the incidence of postoperative delirium was significantly lower with modified perioperative protocols. Under this modified protocol, we reduced or avoided using methyl- 
prednisolone and patients confined to bed were encouraged to move freely while maintaining neck immobilization. We postulate that these changed protocols may help restore normal circadian rhythm, thereby reducing the risk of subsequent delirium.

Disturbance of circadian rhythm is thought to be an important contributing factor to the development of postoperative delirium. Melatonin, a pineal gland hormone, and cortisol are known to play an important role in maintaining a normal circadian rhythm. ${ }^{9,10}$ The former has a role in the normal sleep process and its secretion is known to decrease with age. In contrast, a rise in cortisol secretion is known to occur in the awakening phase. Thus, we suggest that the administration of high-dose methylprednisolone, a synthesized glucocorticoid, and age-related decreased secretions of melatonin, may influence the circadian rhythm. ${ }^{11-13}$ In addition, cervical postoperative patients were often confused due to deprived external information sources including visual and auditory stimuli as a result of neck immobilization and age-related hearing loss. In fact, Stein and Thienhaus reported that patients with hearing impairment showed poor speech discrimination and suggested that hearing impairment is a potential risk factor in the development of psychosis in the elderly. ${ }^{14}$ This confusion may contribute to the risk of delirium as rapid resolution was observed, regardless of severity, after restoration of circadian rhythm by mobilization. Limiting glucocorticoid infusion doses and rapid restoration of circadian rhythm after surgery are therefore important for both the prevention and treatment of postoperative delirium in cervical surgery patients.

Although we could not specify its relationship with other organic medical and psychological factors in the occurrence of postcervical spine surgery delirium, possible factors (neurotransmitters, cytokines) have been noted in previous reports. Acetylcholine, a novel neurotransmitter, and anticolinergic activity are known to be associated with the occurrence of delirium. ${ }^{15} \mathrm{With}$ postcardiotomy, postelectroconvulsive, and elderly medical inpatients, the association between serum anticholinergic activity and delirium has been reported. In addition, higher incidence rates of delirium were reported in patients who had received a large amount of anticholinergics. As for dopamine, it is known to interact with acetylcholine and is upregulated in delirium states and therapeutic effect of Haloperidol, a dopamine blocker on delirium suggests that dopamine may have an indirect effect on delirium via its anticholinergic activity. According to previous studies, cytokines (IL6, IL8, etc.), are known to cause delirium by CNS neuronal stress. ${ }^{16}$ Since surgery induced inflammation may affect cytokine activity, these mechanisms might be underlying factors in postoperative delirium.

A limitation of the methodology in the present study is that hearing impairment was diagnosed when patients had difficulty repeating words spoken in a conversation voice by the head nurse. Generally, an assessment of the degree of hearing loss is based on frequency averages at 500,1000 , and $200 \mathrm{~Hz}$ (the main speech frequency) in the elderly people. ${ }^{17,18}$ When patients had difficulty repeating words spoken in a conversation voice by an observer standing behind the patients, the patients would be diagnosed as hearing impairment likely to benefit from an aid. ${ }^{17}$ We thus believe that the simple screening test used in the present study could have, if not precisely, discriminated hearing impairment.

Since a state of delirium can possibly bring about unexpected traumatic conditions to the spine and spinal cord, preventing such a state is important for postoperative management of cervical spine surgery. Judging from our results, early postoperative free movement by wearing of cervical orthosis and reduced usage of methylprednisolone could be effective in preventing postoperative delirium. In addition, the usage of various types of medication such as melatonin, haloperidol, donepezil, risperidone, and benzodiazepines are effective in preventing postoperative delirium. ${ }^{19-23}$ All of these treatments, including our delirium prevention policy (with the exception of haloperidol therapy) aim to normalize the circadian rhythm from a disordered state caused by surgery-related multifactors. We thus believe that developing intensive perioperative therapeutic protocols in accordance with these concepts will prevent the occurrence of postoperative delirium in high-risk groups.

\section{- Key Points}

- Incidence and clinical features of postoperative delirium in cervical spine surgeries were retrospectively analyzed.

- Age over 70, hearing impairments and usage of high-dose methylprednisolone were associated with incidence of postoperative delirium.

- Modified postoperative protocol reduced incidence of postoperative delirium.

\section{Acknowledgments}

The authors would like to express their gratitude to Professor S. Komune for scientific advice, and Ms. H. Taki for technical assistance.

\section{References}

1. Inouye SK. Delirium in older persons. N Engl J Med 2006;354:1157-65.

2. Bruce AJ, Ritchie CW, Blizard R, et al. The incidence of delirium associated with orthopaedic surgery: a meta-analytic review. Int Psychogeriatr 2007; 19:197-214.

3. Dyer CB, Ashton CM, Teasdale TA. Postoperative delirium: a review of 80 primary data-collection studies. Arch Intern Med. 1995;155:461-5.

4. Gao R, Yang ZZ, Li M, et al. Probable risk factors for postoperative delirium in patients undergoing spinal surgery. Eur Spine J 2008;17:1531-7.

5. Kawaguchi Y, Kanamori M, Ishihara H, et al. Postoperative delirium in spine surgery. Spine J 2006;6:164-9.

6. Santos FS, Velasco IT, Fráguas R Jr. Risk factors for delirium in the elderly after coronary artery bypass graft surgery. Int Psychogeriatr 2004;16:175-93.

7. Jeong JW, Kim KW, Lee DY, et al. A normative study of the Revised Hasegawa Dementia Scale: comparison of demographic influences between the Revised Hasegawa Dementia Scale and the Mini-Mental Status Examination. Dement Geriatr Cogn Disord 2007;24:288-93. 
8. Gemert van LA, Schuurmans MJ. The Neecham Confusion Scale and the Delirium Observation Screening Scale: capacity to discriminate and ease of use in clinical practice. BMC Nurs 2007;6:3.

9. James FO, Cermakian N, Boivin DB. Circadian rhythms of melatonin, cortisol, and clock gene expression during simulated night shift work. Sleep 2007;30:1427-36.

10. Uchida K, Aoki T, Ishizuka B. Postoperative delirium and plasma melatonin. Med Hypotheses 1999;53:103-6.

11. Campbell KM, Schubert DS. Delirium after cessation of glucocorticoid therapy. Gen Hosp Psychiatry 1991;13:270-2.

12. O'Keeffe ST, Devlin JG. Delirium and the dexamethasone suppression test in the elderly. Neuropsychobiology 1994;30:153-6.

13. Shigeta H, Yasui A, Nimura Y, et al. Postoperative delirium and melatonin levels in elderly patients. Am J Surg 2001;182:449-54.

14. Stein LM, Thienhaus OJ. Hearing impairment and psychosis. Int Psychogeriatr 1993;5:49-56.

15. Tune LE, Egeli S. Acetylcholine and delirium. Dement Geriatr Cogn Disord $1999 ; 10: 342-4$.
16. de Rooij SE, van Munster BC, Korevaar JC, et al. Cytokines and acute phase response in delirium. J Psychosom Res 2007;62:521-5.

17. Macphee GJ, Crowther JA, McAlpine CH. A simple screening test for hearing impairment in elderly patients. Age Ageing 1988;17:347-51.

18. Baraldi Gdos S, de Almeida LC, Borges AC. Hearing loss in aging. Braz J Otorhinolaryngol 2007;73:58-64.

19. Hanania M, Kitain E. Melatonin for treatment and prevention of postoperative delirium. Anesth Analg 2002;94:338-9.

20. Seitz D, Gill SS. Perioperative haloperidol to prevent postoperative delirium. J Am Geriatr Soc 2006;54:861; author reply 861-3.

21. Liptzin B, Laki A, Garb JL, et al. Donepezil in the prevention and treatment of post-surgical delirium. Am J Geriatr Psychiatry 2005;13:1100-6.

22. Prakanrattana U, Prapaitrakool S. Efficacy of risperidone for prevention of postoperative delirium in cardiac surgery. Anaesth Intensive Care 2007;35: 714-9.

23. Aizawa KT, Kanai Y, Saikawa Y, et al. A novel approach to the prevention of postoperative delirium in the elderly after gastrointestinal surgery. Surg Today 2002;32:310-4. 